\title{
On the determinant of quaternionic polynomial matrices and its application to system stability
}

\author{
Ricardo Pereira* and Paula Rocha*
}

\begin{abstract}
SUMMARY
In this paper we propose a definition of determinant for quaternionic polynomial matrices inspired by the well-known Dieudonné determinant for the constant case. This notion allows to characterize the stability of linear dynamical systems with quaternionic coefficients, yielding results which generalize the ones obtained for the real and complex cases.
\end{abstract}

\section{Introduction}

The research reported in this paper is motivated by the study of stability for linear dynamical systems with quaternionic coefficients. These systems can be used to model several physical phenomena, for instance, in areas such as robotics and quantum mechanics. More concretely, quaternions are a powerful tool in the description of rotations [1]. There are situations, especially in robotics, where the rotation of a rigid body depends on time, and this dynamics is advantageously written in terms of quaternionic differential or difference equations. The effort to control the rotation dynamics motivates the study of these equations from a system theoretic point of view (see, for instance, [2]). Another motivation stems from quantum mechanics, where a quaternionic formulation of the Schrödinger equation has been proposed in the sixties along with experiments to check the existence of quaternionic potentials (see, for instance, [3]). This theory leads to differential equations with quaternionic coefficients [4].

A common way to treat linear dynamical systems is to consider state space models. The stability of a linear state space system $\dot{x}=A x$ with real or complex coefficients is essentially characterized by the location of the eigenvalues of the system matrix $A$, involving thus the computation of the determinant of the polynomial matrix $s I-A[5]$. In a more general setting, the growth of the solutions (or trajectories) of a linear higher order differential equation with constant (square) matrix coefficients $\left(R_{m} \frac{d^{m}}{d t^{m}}+\cdots+R_{1} \frac{d}{d t}+R_{0}\right) w=0$ can also be characterized in terms of the zeros of the determinant of the polynomial matrix $R(s):=R_{m} s^{m}+\cdots+R_{1} s+R_{0}$ [6].

*Department of Mathematics, University of Aveiro, 3810-193 Portugal (ricardo@mat.ua.pt, procha@mat.ua.pt) 
When trying to generalize these results to the quaternionic case we were confronted with the lack of a notion of determinant for quaternionic polynomial matrices. Indeed, due to the non-commutativity of the field of quaternions, the determinant of quaternionic matrices cannot be defined as in the commutative (e.g., real or complex) case. Several definitions have been proposed for matrices over the quaternionic skew field [7, 8], for instance, the Study determinant [9] (in 1920) and latter, in the forties, the Dieudonné determinant [10]. Whereas the former is defined in terms of complex adjoint matrices, the latter results from a direct approach that remains entirely at the quaternionic level. However, up to our knowledge, this work has not been extended to the polynomial ring case.

Considering complex adjoints for quaternionic polynomial matrices, as introduced in [11], the Study determinant can be generalized in a straightforward manner. However, the natural question arises whether it is possible to define a polynomial determinant without leaving the quaternionic framework, as happens with the Dieudonné determinant for the constant case. In this paper we give a positive answer to this question by introducing the polynomial determinant Pdet.

Further, we prove that, as should be expected, the zeros of $\operatorname{Pdet}(s I-A)$ are precisely the (right) eigenvalues of the quaternionic matrix $A$, which allows to generalize the stability analysis of linear dynamical systems to the quaternionic case.

\section{Preliminaries}

\subsection{Stability of linear systems}

Systems described by linear differential equations with constant matrix coefficients

$$
\left(R_{m} \frac{d^{m}}{d t^{m}}+\cdots+R_{1} \frac{d}{d t}+R_{0}\right) w=0
$$

with $R(s):=R_{m} s^{m}+\cdots+R_{1} s+R_{0} \in \mathbb{F}^{n \times n}[s]$ and $\mathbb{F}$ the field $\mathbb{R}$ or $\mathbb{C}$, can be considered as a generalization of state space systems

$$
\dot{x}=A x .
$$

Such systems have been widely studied within the behavioral approach to dynamical systems introduced by J. C. Willems $[6,12]$ with respect to various aspects. Here we are particularly interested in the question of stability.

A linear system is said to be (asymptotically) stable if all its solutions tend to zero as time goes to infinity. For systems described by an equation of the type (2.2), stability is characterized by the following proposition.

Proposition 2.1. [5] The state system (2.2) is stable if and only if $\operatorname{det}(\lambda I-A) \neq 0$, $\forall \lambda \in \mathbb{C}_{0}^{+}$, where $\mathbb{C}_{0}^{+}:=\{z \in \mathbb{C}: \operatorname{Re}(z) \geq 0\}$

This result also generalizes to systems with description (2.1), as stated bellow. 
Proposition 2.2. [6] The system (2.1) is stable if and only if $\operatorname{det} R(\lambda) \neq 0, \forall \lambda \in$ $\mathbb{C}_{0}^{+}$.

In this paper we replace the field $\mathbb{F}$ by the quaternionic skew field $\mathbb{H}$, to be properly defined in the next section. This gives rise to linear quaternionic systems, with trajectories evolving over $\mathbb{H}^{n}$.

In order to study the stability of such systems we first introduce some preliminary concepts on quaternions and quaternionic polynomials and then, in Section 3, our notion of determinant for quaternionic polynomial matrices.

\subsection{The quaternionic skew field}

The set

$$
\mathbb{H}=\{a+b \mathbf{i}+c \mathbf{j}+d \mathbf{k}: a, b, c, d \in \mathbb{R}\},
$$

where the imaginary units $\mathbf{i}, \mathbf{j}$, $\mathbf{k}$ satisfy $\mathbf{i}^{2}=\mathbf{j}^{2}=\mathbf{k}^{2}=\mathbf{i j} \mathbf{k}=-1$ and, consequently,

$$
\mathrm{ij}=\mathrm{k}=-\mathrm{ji}, \quad \mathbf{j k}=\mathbf{i}=-\mathbf{k j}, \quad \mathbf{k i}=\mathbf{j}=-\mathbf{i k},
$$

is an associative but noncommutative division algebra over $\mathbb{R}$ called quaternionic skew field. The real and imaginary parts of a quaternion $\eta=a+b \mathbf{i}+c \mathbf{j}+d \mathbf{k}$ are defined as $\operatorname{Re} \eta=a$ and $\operatorname{Im} \eta=b \mathbf{i}+c \mathbf{j}+d \mathbf{k}$, respectively, whereas, similar to the complex case, the conjugate $\bar{\eta}$ is given by $\bar{\eta}=a-b \mathbf{i}-c \mathbf{j}-d \mathbf{k}$ and the norm $|\eta|$ is defined as $|\eta|=\sqrt{\eta \bar{\eta}}$.

Two quaternions $\eta$ and $\nu$ are said to be similar, $\eta \sim \nu$, if there exists a nonzero $\alpha \in \mathbb{H}$ such that $\eta=\alpha \nu \alpha^{-1}$. Similarity is an equivalence relation and we denote by $[\nu]$ the equivalence class containing $\nu$. Note that $\bar{\nu} \in[\nu]$, as a consequence of the following theorem.

Theorem 2.3. [13] Given two quaternions $\eta, \nu \in \mathbb{H}, \eta \in[\nu]$ if and only if $\operatorname{Re} \eta=$ $\operatorname{Re} \nu$ and $|\eta|=|\nu|$.

This theorem also implies that every quaternion is similar to a complex number. Indeed, if $\eta=a+b \mathbf{i}+c \mathbf{j}+d \mathbf{k} \in \mathbb{H}$ then the complex $z=a+\sqrt{b^{2}+c^{2}+d^{2}} \mathbf{i} \in \mathbb{C}$ is similar to $\eta$ since $\operatorname{Re} z=\operatorname{Re} \eta$ and $|z|=|\eta|$.

\subsection{Quaternionic polynomials}

In this section we define the quaternionic polynomials to be considered in the sequel and study some of their most relevant properties for our purposes. Unlike the real or complex case, there are several possible ways to define quaternionic polynomials since the coefficients can be taken to be on the right, on the left or on both sides of the indeterminate (see, e.g., [14]). In this paper we shall adopt the following definition. 
Definition 2.4. A quaternionic polynomial $p(s)$ is defined as

$$
p(s)=p_{n} s^{n}+\cdots+p_{1} s+p_{0}, p_{l} \in \mathbb{H}, l=1, \ldots, n .
$$

If $p_{n} \neq 0$, the degree of the quaternionic polynomial $p(s), \operatorname{deg} p(s)$, is $n$ and its leading coefficient, lc $p(s)$, is $p_{n}$. As usual, if lc $p(s)=1, p(s)$ is said to be monic.

We denoted by $\mathbb{H}[s]$ the set of quaternionic polynomials endowed with the following sum and product. Given two quaternionic polynomials $p(s)=\sum_{n=0}^{N} p_{n} s^{n}$ and $q(s)=$ $\sum_{m=0}^{M} q_{m} s^{m}$ :

$$
\begin{gathered}
p(s)+q(s)=\sum_{l=0}^{\max \{N, M\}}\left(p_{l}+q_{l}\right) s^{l}, \\
p(s) q(s)=\sum_{l=0}^{N+M} \sum_{n+m=l} p_{n} q_{m} s^{l}=: p q(s) .
\end{gathered}
$$

Clearly, $\mathbb{H}[s]$ is a noncommutative ring [15]. Note moreover that, with the defined operations, unlike the commutative case, evaluation of polynomials is not a ring homomorphism, i.e., if $r=p q \in \mathbb{H}[s]$, then in general $r(\lambda) \neq p(\lambda) q(\lambda), \lambda \in \mathbb{H}$, as shown in the following example.

Example 2.5. Let $p(s)=s-\mathbf{i}, q(s)=s-\mathbf{j}$ and

$$
r(s)=p(s) q(s)=(s-\mathbf{i})(s-\mathbf{j})=s^{2}-(\mathbf{i}+\mathbf{j}) s+\mathbf{k} .
$$

Then

$$
r(\mathbf{i})=\mathbf{i}^{2}-(\mathbf{i}+\mathbf{j}) \mathbf{i}-\mathbf{k}=2 \mathbf{k} \quad \text { but } \quad p(\mathbf{i}) q(\mathbf{i})=(\mathbf{i}-\mathbf{i})(\mathbf{i}-\mathbf{j})=0
$$

To simplify the notation, we may omit the indeterminate and write $p \in \mathbb{H}[s]$, if no ambiguity arises.

A polynomial $p(s)$ is said to be an invertible element or a unit of $\mathbb{H}[s]$ if there exists $q(s) \in \mathbb{H}[s]$ such that $q(s) p(s)=p(s) q(s)=1$. This clearly means that $p(s)$ must be a nonzero quaternionic constant.

Conjugacy is extended to quaternionic polynomials by linearity and by the rule $\overline{a s^{n}}=\bar{a} s^{n}, \forall a \in \mathbb{H}$. As a consequence, $\overline{p q}=\bar{q} \bar{p}$ for every $p, q \in \mathbb{H}[s]$ (see [11]). Moreover, the following result holds.

Proposition 2.6. [11] Let $p, q \in \mathbb{H}[s]$. Then

(i) $p \bar{p}=\bar{p} p \in \mathbb{R}[s]$.

(ii) If $p q \in \mathbb{R}[s]$, then $p q=q p$. 
Remark 2.7. Note that in the particular case where $p$ is monic and of degree 1 , i.e., $p(s)=s-\alpha, \alpha \in \mathbb{H}$, the product $p \bar{p}$ is an invariant of the similarity class of $\alpha$. Indeed, $p \bar{p}=(s-\alpha)(s-\bar{\alpha})=s^{2}-2(\operatorname{Re} \alpha) s+|\alpha|^{2}$. Since, by Theorem 2.3, for all $\alpha^{\prime} \sim \alpha$ we have that $\operatorname{Re} \alpha=\operatorname{Re} \alpha^{\prime}$ and $|\alpha|=\left|\alpha^{\prime}\right|$ it is clear that if $q(s)=s-\alpha^{\prime}$, with $\alpha \sim \alpha^{\prime}$, then $p \bar{p}=q \bar{q}$.

A quaternionic polynomial $d(s)$ is said to be a left divisor of a polynomial $p(s) \in$ $\mathbb{H}[s]$, which we shall denote by $\left.d(s)\right|_{l} p(s)$, or, equivalently, $p(s)$ is said to be a right multiple of $d(s)$, if there exists a polynomial $q(s)$ such that

$$
p(s)=d(s) q(s) .
$$

If $d(s)$ is a left divisor of both $p(s)$ and $q(s)$, and $d(s)$ is a right multiple of every common left divisor of $p(s)$ and $q(s)$, then $d(s)$ is a greatest common left divisor (gcld) of $p(s)$ and $q(s)$. The gcld is unique up to right multiplication by a unit. Two polynomials $p(s)$ and $q(s)$ are called left coprime if every gcld of $p(s)$ and $q(s)$ is a unit.

The definitions of right divisor, gcrd, and right coprimeness (left multiple) are entirely analogous. We shall use the notation $\left.d(s)\right|_{r} p(s)$ to indicate that $d(s)$ is a right divisor of $p(s)$.

In general, the gcld's of two quaternionic polynomials are different from their gcrd's.

Example 2.8. Let $p(s)=\mathbf{j} s-\mathbf{k}$ and $q(s)=-\mathbf{i} s+1$. Then every $\operatorname{gcrd}(p(s), q(s))$ is of the form $\eta(-\mathbf{i} s+1), \eta \in \mathbb{H}$, and every $\operatorname{gcld}(p(s), q(s))$ is a constant $\nu \in \mathbb{H}$. $\diamond$

The zeros of a quaternionic polynomial $p \in \mathbb{H}[s]$ are the values $\lambda \in \mathbb{H}$ such that $p(\lambda)=0$. The problem of finding such zeros as well as the study of the fundamental theorem of algebra for the quaternionic case were first addressed in the forties by Niven and Eilenberg $[16,17]$. This was followed by other contributions, such as $[18$, 19], where the questions of finding and counting the number of zeros of quaternionic polynomials have been investigated.

A pair $(p, q) \in \mathbb{H}[s]^{2}$ is zero coprime if $p$ and $q$ do not have common zeros. Factors of a polynomial are usually related to its zeros, but the fact that evaluation is not a ring homomorphism, as mentioned before, implies that the relation between the factors and the zeros of a quaternionic polynomial is not as simple as for real or complex polynomials. Results concerning this nontrivial relation can be found in [20, 21].

The next proposition establishes a connection between zeros and right divisors.

Proposition 2.9. [21] A quaternion $\alpha$ is a zero of a nonzero $p \in \mathbb{H}[s]$ if and only if the polynomial $s-\alpha$ is a right divisor of $p$.

Note that this implies that zero coprimeness is equivalent to right coprimeness. However, if $d(s)$ is a left divisor of $p(s)$, the zeros of $d(s)$ are not necessarily zeros of $p(s)$. For instance, in Example 2.5, $p(s)=(s-\mathbf{i})$ is a left divisor of $r(s), p(\mathbf{i})=0$ but $r(\mathbf{i})=2 \mathbf{k} \neq 0$. 
Nevertheless, there is still some connection between the zeros of a polynomial and the zeros of its left divisors. Indeed, let $r=p q \in \mathbb{H}[s]$, if $\alpha \in \mathbb{H}$ is a zero of the polynomial $r$ but not of its right divisor $q$, then its left divisor $p$ must have a zero that is equivalent to $\alpha$. This is formalized in the following result.

Proposition 2.10. [21] Let $r=p q \in \mathbb{H}[s]$ and $\alpha \in \mathbb{H}$ be such that $\beta=q(\alpha) \neq 0$. Then

$$
r(\alpha)=p\left(\beta \alpha \beta^{-1}\right) q(\alpha) .
$$

In particular, $\alpha$ is a zero of $r$ if and only if $\beta \alpha \beta^{-1}$ is a zero of $p$.

Besides the similarity concept that we have been using up to this point, a second notion will play an important role in the sequel. In order to distinguish it from the first one, we shall call it $J$-similarity, and denote it by $\sim_{J}$, where the $J$ stands for Jacobson, who first introduced this notion, [15].

Definition 2.11. [22, 15] Two quaternionic polynomials $a, d \in \mathbb{H}[s]$ are said to be $J$-similar, $a \sim_{J} d$, if there exist $b, c \in \mathbb{H}[s]$ such that the relation

$$
a b=c d
$$

is a coprime relation. By this it is meant that $(a, c)$ are left coprime and $(b, d)$ are right coprime.

Our next result, that will be relevant in Section 3, relates the real polynomials $a \bar{a}$ and $d \bar{d}$ in case $a \sim_{J} d$.

Proposition 2.12. Let $a=\sum_{l=0}^{n} a_{l} s^{l}, d=\sum_{l=0}^{m} d_{l} s^{l} \in \mathbb{H}[s]$ be such that $\left|a_{n}\right|=\left|d_{m}\right|$.

If $a \sim_{J} d$, i.e., there exists $b, c \in \mathbb{H}[s]$ such that

$$
a b=c d
$$

is a coprime relation, then

$$
a \bar{a}=d \bar{d} \quad \text { and } \quad b \bar{b}=c \bar{c} .
$$

Proof. See Appendix A.

\subsection{Quaternionic polynomial matrices}

As usual, $\mathbb{H}^{g \times r}$ and $\mathbb{H}^{g \times r}[s]$ will respectively denote the set of the $g \times r$ matrices with entries in $\mathbb{H}$ and in $\mathbb{H}[s]$. As for polynomials, for simplicity, we may also omit the indeterminate $s$ and write $R \in \mathbb{H}^{g \times r}[s]$ if no ambiguity arises.

A square matrix $R \in \mathbb{H}^{g \times g}$ has full rank if for $X \in \mathbb{H}^{1 \times g}, X R=0$ implies $X=0$. The same definition holds for polynomial matrices. 
A matrix $U \in \mathbb{H}^{g \times g}[s]$ is said to be a unimodular polynomial matrix if there exists a polynomial matrix $V \in \mathbb{H}^{g \times g}[s]$ such that $V U=U V=I$, where $I$ is the identity matrix.

According to [7] we shall use the following notation.

Notation 2.13. Denote by $P_{l m}$ the matrix that is obtained from the identity by interchanging the $l^{t h}$ and $m^{\text {th }}$ rows. Denote by $B_{l m}(\alpha)$, where $\alpha \in \mathcal{C}$ and $\mathcal{C}$ is a set, the matrix that is obtained from the identity by adding the $m^{\text {th }}$ row multiplied by $\alpha$ to the $l^{\text {th }}$ row. Finally denote by $S L(n, \mathcal{C})$ the set of all $n \times n$ matrices that can be decomposed as a product of matrices of the types $P_{l m}$ and $B_{l m}(\alpha), \alpha \in \mathcal{C}$.

As in the commutative case, it is possible to obtain a triangular matrix pre-multiplying the original one by a matrix belonging to $S L(n, \mathbb{H}[s])$. The result is formalized next. The proof is completely analogous to the one of [6, Theorem B.1.1] and is based in the Euclidian division algorithm.

Lemma 2.14. For every $R \in \mathbb{H}^{n \times n}[s]$ there exists a matrix $U \in S L(n, \mathbb{H}[s])$ such that

$$
U R=T,
$$

where $T \in \mathbb{H}^{n \times n}[s]$ is a triangular matrix.

The following result is relevant for the definition of the determinant of quaternionic polynomial matrices that will be given in Section 3 .

Lemma 2.15. Let

$$
R=\left[\begin{array}{l}
\gamma_{1} \\
\gamma_{2}
\end{array}\right] \in \mathbb{H}^{2 \times 1}[s] .
$$

Then there exists $U \in S L(n, \mathbb{H}[s])$ such that

$$
U R=\left[\begin{array}{ll}
u_{11} & u_{12} \\
u_{21} & u_{22}
\end{array}\right]\left[\begin{array}{l}
\gamma_{1} \\
\gamma_{2}
\end{array}\right]=\left[\begin{array}{l}
t \\
0
\end{array}\right]
$$

with $t$ a gcrd of $\gamma_{1}$ and $\gamma_{2}$, and letting $g \in \mathbb{H}[s]$ be such that $\gamma_{2}=g t$,

$$
u_{21} \sim_{J} g \quad \text { and } \quad \mid \text { lc } u_{21}|=| \operatorname{lc} g \mid .
$$

Proof. See Appendix A.

\section{Polynomial determinant}

Before considering the polynomial case, it should be noticed that the question of defining a determinant for constant quaternionic matrices is itself nontrivial and has deserved the attention of several mathematicians throughout the years.

Indeed, due to the noncommutativity of the quaternionic skew-field $\mathbb{H}$, it is not possible to extend the usual definition of determinant. For instance, let

$$
A=\left[\begin{array}{ll}
\mathbf{i} & 0 \\
0 & \mathbf{j}
\end{array}\right]
$$


and suppose that the usual properties for determinants of complex matrices would hold. Then

$$
\operatorname{det} A=\operatorname{det}\left[\begin{array}{ll}
\mathbf{i} & 0 \\
0 & \mathbf{j}
\end{array}\right]=\mathbf{i} \operatorname{det}\left[\begin{array}{ll}
1 & 0 \\
0 & \mathbf{j}
\end{array}\right]=\mathbf{i j} \operatorname{det}\left[\begin{array}{ll}
1 & 0 \\
0 & 1
\end{array}\right]=\mathbf{i j}=\mathbf{k}
$$

whereas, on the other hand

$$
\operatorname{det} A=\operatorname{det}\left[\begin{array}{ll}
\mathbf{i} & 0 \\
0 & \mathbf{j}
\end{array}\right]=\mathbf{j} \operatorname{det}\left[\begin{array}{ll}
\mathbf{i} & 0 \\
0 & 1
\end{array}\right]=\mathbf{j i} \operatorname{det}\left[\begin{array}{ll}
1 & 0 \\
0 & 1
\end{array}\right]=\mathbf{j i}=-\mathbf{k}
$$

leading to an absurd.

The first mathematician who tried to define the determinant of a quaternionic matrix was Arthur Cayley in 1845 [23], but his definition was not satisfactory (see [7]). Only in the twentieth century new developments in this topic were achieved. Moore [24] showed that the Cayley determinant makes sense when restricted to hermitian quaternionic matrices and some different, but closely related, definitions such as the determinants of Study [9] and Dieudonné [10] were given. More recently, in the nineties, Gelfand and Retakh [25] introduced the notion of quasideterminant, but this is beyond the scope of this article.

The determinants of Study and Dieudonné are in accordance with the following definition of determinant for quaternionic matrices, that can be regarded as a generalization of the notion of determinant for the real and complex cases.

Definition 3.1. [7] A function $d: \mathbb{H}^{n \times n} \rightarrow \mathbb{H}$ is said to be a determinant if it satisfies the following axioms:

(i) $d(A)=0$ if and only if $A$ has not full rank.

(ii) $d(A B)=d(A) d(B)$ for all $A, B \in \mathbb{H}^{n \times n}$.

(iii) If $A^{\prime}=B_{l m}(\alpha) A, \alpha \in \mathbb{H}$, then $d\left(A^{\prime}\right)=d(A)$.

We shall also adopt this definition for the polynomial case and say that $d: \mathbb{H}^{n \times n}[s] \rightarrow$ $\mathbb{H}[s]$ is a polynomial determinant if it satisfies the conditions of Definition 3.1, with $\mathbb{H}$ replaced by $\mathbb{H}[s]$.

The concrete notion of polynomial determinant that we propose is motivated by the definition of Dieudonné determinant given below. First we state an auxiliary lemma.

Lemma 3.2. [10] Let $A \in \mathbb{H}^{n \times n}$ be invertible. Then there exists a matrix $U \in$ $S L(n, \mathbb{H})$ such that

$$
U A=\operatorname{diag}(1, \ldots, 1, \alpha), \alpha \in \mathbb{H} .
$$

Definition 3.3. [10] Let $A \in \mathbb{H}^{n \times n}$; the Dieudonné determinant of $A$, denoted by $\operatorname{Ddet}(A)$, is defined as follows.

- If $A$ has not full rank, then $\operatorname{Ddet}(A):=0$. 
- Otherwise, let $U \in S L(n, \mathbb{H})$ be such that

$$
U A=\operatorname{diag}(1, \ldots, 1, \alpha), \alpha \in \mathbb{H} .
$$

Then $\operatorname{Ddet}(A):=|\alpha|$.

In [10], Dieudonné shows that Ddet (also called normalized Dieudonné determinant in [7]) is equivalent to his determinant originally defined in more abstract terms.

Remark 3.4. Note that the Dieudonné determinant is not an extension of the determinant of real matrices, i.e., given a real matrix $A \in \mathbb{R}^{n \times n}$ then, in general, $\operatorname{Ddet}(A) \neq \operatorname{det}(A)$. For instance, consider the simple scalar case $A=-1$. Then $\operatorname{det}(A)=-1$ but $\operatorname{Ddet}(A)=|-1|=1$.

The straightforward extension of the Dieudonné determinant to the polynomial case faces two major difficulties. First it is impossible to diagonalize a polynomial matrix $R \in \mathbb{H}^{n \times n}[s]$ as in Lemma 3.2, i.e., only multiplying on the left by a matrix $U \in$ $S L(n, \mathbb{H}[s])$. Second it does not make sense to define the norm of a polynomial.

However, as seen in Lemma 2.14, given a matrix $R \in \mathbb{H}^{n \times n}[s]$ there exists a matrix $U \in S L(n, \mathbb{H}[s])$ such that $U R=T$, where $T$ is a triangular polynomial matrix. Using an approach in some sense similar to the one of Dieudonné, we define a polynomial determinant for the quaternionic polynomial matrix $R$ with basis on the diagonal elements of the triangular matrix $T$.

Definition 3.5. We define the function $\operatorname{Pdet}(\cdot): \mathbb{H}^{n \times n}[s] \rightarrow \mathbb{R}[s]$ as follows.

Let $R \in \mathbb{H}^{n \times n}[s]$. Let further $U \in S L(n, \mathbb{H}[s])$ be such that $U R$ is upper triangular, i.e.,

$$
U R=T=\left[\begin{array}{ccccc}
\gamma_{1} & * & \cdots & * & * \\
0 & \gamma_{2} & \ddots & \vdots & \vdots \\
0 & 0 & \ddots & * & * \\
\vdots & \vdots & \ddots & \gamma_{n-1} & * \\
0 & 0 & \cdots & 0 & \gamma_{n}
\end{array}\right]
$$

Then

$$
\operatorname{Pdet}(R):=\prod_{l=1}^{n} \gamma_{l} \bar{\gamma}_{l}
$$

Note that Definition 3.5 is well posed as a consequence of the following Lemma.

Lemma 3.6. Given $R \in \mathbb{H}^{n \times n}[s]$, let $T$ and $T^{\prime}$ be two triangular matrices obtained by pre-multiplying $R$ by $U$ and $U^{\prime} \in S L(n, \mathbb{H}[s])$, respectively. Let further $\gamma_{1}, \ldots, \gamma_{n}$ and $\gamma_{1}^{\prime}, \ldots, \gamma_{n}^{\prime}$ be the elements of the main diagonal of $T$ and $T^{\prime}$, respectively. Then

$$
\prod_{l=1}^{n} \gamma_{l}^{\prime} \bar{\gamma}_{l}^{\prime}=\prod_{l=1}^{n} \gamma_{l} \bar{\gamma}_{l} .
$$


Proof. If $R$ has not full rank, the same happens for every triangular matrix $\widetilde{T}$ such that $U R=\widetilde{T}$, for some $U \in S L(n, \mathbb{H}[s]$. This clearly implies that at least one of the diagonal elements of $T$ is zero, and the same happens with $T^{\prime}$. Therefore (3.2) holds since both sides of the equality are zero.

Let now $R$ have full rank. Suppose first that $R \in \mathbb{H}^{2 \times 2}[s]$ is triangular and $2 \times 2$, i.e.,

$$
R=\left[\begin{array}{cc}
\gamma_{1} & \gamma_{12} \\
0 & \gamma_{2}
\end{array}\right], \text { with } \gamma_{1}, \gamma_{2} \neq 0
$$

Let $U=\left[\begin{array}{ll}u_{11} & u_{12} \\ u_{21} & u_{22}\end{array}\right] \in S L(2, \mathbb{H}[s])$ be such that

$$
U R=\left[\begin{array}{ll}
u_{11} & u_{12} \\
u_{21} & u_{22}
\end{array}\right]\left[\begin{array}{cc}
\gamma_{1} & \gamma_{12} \\
0 & \gamma_{2}
\end{array}\right]=\left[\begin{array}{ll}
u_{11} \gamma_{1} & u_{11} \gamma_{12}+u_{12} \gamma_{2} \\
u_{21} \gamma_{1} & u_{21} \gamma_{12}+u_{22} \gamma_{2}
\end{array}\right]=\left[\begin{array}{cc}
\gamma_{1}^{\prime} & \gamma_{12}^{\prime} \\
0 & \gamma_{2}^{\prime}
\end{array}\right]=T^{\prime}
$$

Then $u_{21} \gamma_{1}=0$, i.e., $u_{21}=0$ and therefore $U$ is triangular. Taking into account that $U \in S L(2, \mathbb{H}[s])$, this implies that $u_{11}$ and $u_{22}$ are nonzero constants, i.e., $u_{11}, u_{22} \in \mathbb{H} \backslash\{0\}$. We next show that $\left|u_{11}\right|\left|u_{22}\right|=1$. Indeed, it is not difficult to see that there exists $V \in S L(2, \mathbb{H}[s])$ such that

$$
V U=\left[\begin{array}{cc}
1 & 0 \\
0 & u_{11} u_{22}
\end{array}\right]
$$

Since $V U \in S L(2, \mathbb{H}[s])$ and it is a constant matrix, this implies that $V U \in$ $S L(2, \mathbb{H})$. Consequently the Dieudonné determinant of $V U$ must be equal to 1 , i.e.,

$$
\operatorname{Ddet}(V U)=\left|u_{11} u_{22}\right|=\left|u_{11}\right|\left|u_{22}\right|=1 \text {. }
$$

Recall that, as mentioned in Section 2.3, for every $p, q \in \mathbb{H}[s], p \bar{p} \in \mathbb{R}[s]$ and $\bar{p} \bar{q}=\overline{q p}$. Hence,

$$
\gamma_{1}^{\prime}{\overline{\gamma^{\prime}}}_{1} \gamma_{2}^{\prime}{\overline{\gamma^{\prime}}}_{2}=u_{11} \gamma_{1} \bar{\gamma}_{1} \overline{u_{11}} u_{22} \gamma_{2} \bar{\gamma}_{2} \overline{u_{22}}=\gamma_{1} \bar{\gamma}_{1} \gamma_{2} \bar{\gamma}_{2}\left|u_{11}\right|^{2}\left|u_{22}\right|^{2}=\gamma_{1} \bar{\gamma}_{1} \gamma_{2} \bar{\gamma}_{2}
$$

If $R \in \mathbb{H}^{n \times n}[s]$ is triangular and $U \in S L(n, \mathbb{H}[s])$ is such that $U R=T^{\prime}$, with $T^{\prime}$ triangular, analogously to the previous case the matrix $U$ must also be triangular and the product of the norms of its main diagonal elements is equal to 1 . Thus, the equality (3.2) holds.

Finally, consider the case where $R$ is not triangular. Let $U, U^{\prime} \in S L(n, \mathbb{H}[s])$ be such that

$$
U R=T, \quad U^{\prime} R=T^{\prime}, \text { with } T, T^{\prime} \text { triangular. }
$$

Then

$$
T^{\prime}=U^{\prime} R=U^{\prime} U^{-1} U R=U^{\prime \prime} T,
$$

where $U^{\prime \prime}=U^{\prime} U^{-1} \in S L(n, \mathbb{H}[s])$, and, by the previous case, we can conclude that the equality (3.2) holds. 
Example 3.7. Let

$$
R(s)=\left[\begin{array}{cc}
(s+2 \mathbf{j})(s+\mathbf{j}) & (s+2 \mathbf{j})(2 s+\mathbf{k})(s+3 \mathbf{i})+2 s+3 \\
s+\mathbf{j} & (2 s+\mathbf{k})(s+3 \mathbf{i})
\end{array}\right] .
$$

Then

$$
R=U T=\left[\begin{array}{cc}
s+2 \mathbf{j} & 1 \\
1 & 0
\end{array}\right]\left[\begin{array}{cc}
s+\mathbf{j} & (2 s+\mathbf{k})(s+3 \mathbf{i}) \\
0 & 2 s+3
\end{array}\right],
$$

and $U \in S L(n, \mathbb{H}[s])$. Therefore

$$
\operatorname{Pdet}(R)=(s+\mathbf{j})(\overline{s+\mathbf{j}})(2 s+3)(\overline{2 s+3})=\left(s^{2}+1\right)(2 s+3)^{2} .
$$

We next show that Pdet(.) is indeed a polynomial determinant. For that purpose we first prove an auxiliary result that states that it is invariant with respect to post-multiplications by a matrix $U \in S L(n, \mathbb{H}[s])$.

Lemma 3.8. Let $M \in \mathbb{H}^{n \times n}[s]$ and $U \in S L(n, \mathbb{H}[s])$. Then

$$
\operatorname{Pdet}(M U)=\operatorname{Pdet}(M) \text {. }
$$

Proof. Since $U \in S L(n, \mathbb{H}[s])$ is a finite product of $P_{l m}$ and $B_{l m}(\alpha)$ matrices, $\alpha \in$ $\mathbb{H}[s]$, it is clearly enough to prove that

$$
\operatorname{Pdet}(M S)=\operatorname{Pdet}(M)
$$

if $S$ is $P_{l m}$ or $B_{l m}(\alpha)$ matrix, $\alpha \in \mathbb{H}[s]$.

If $M$ has not full rank, the equality (3.3) trivially holds.

If $M$ has full rank, there exists a matrix $V \in S L(n, \mathbb{H}[s])$ such that $T=V M$, where $T$ is a triangular matrix, and consequently $\operatorname{Pdet}(M)=\operatorname{Pdet}(T)$. Thus

$$
P \operatorname{det}(M S)=\operatorname{Pdet}(V M S)=\operatorname{Pdet}(T S)
$$

and it is therefore sufficient to show that $\operatorname{Pdet}(T S)=\operatorname{Pdet}(S)$.

Suppose first that $T \in \mathbb{H}^{2 \times 2}[s]$ and is given by

$$
T=\left[\begin{array}{cc}
\gamma_{1} & \gamma_{12} \\
0 & \gamma_{2}
\end{array}\right], \text { with } \gamma_{1}, \gamma_{2} \neq 0, \quad \text { and hence } \quad \operatorname{Pdet}(T)=\gamma_{1} \bar{\gamma}_{1} \gamma_{2} \bar{\gamma}_{2}
$$

In the sequel we show that $\operatorname{Pdet}(T S)=\operatorname{Pdet}(T)$, where $S=B_{12}(\alpha), \alpha \in \mathbb{H}[s]$, or $S=P_{12}$. Note that it is not necessary to prove that $\operatorname{Pdet}(T S)=\operatorname{Pdet}(T)$ with $S=B_{21}(\alpha)$ since $B_{21}(\alpha)=P_{12} B_{12}(\alpha) P_{12}$. The first case is obvious because

$$
T B_{12}(\alpha)=\left[\begin{array}{cc}
\gamma_{1} & \gamma_{12} \\
0 & \gamma_{2}
\end{array}\right]\left[\begin{array}{cc}
1 & \alpha \\
0 & 1
\end{array}\right]=\left[\begin{array}{cc}
\gamma_{1} & \gamma_{1} \alpha+\gamma_{12} \\
0 & \gamma_{2}
\end{array}\right]
$$

On the other hand,

$$
T P_{12}=\left[\begin{array}{cc}
\gamma_{1} & \gamma_{12} \\
0 & \gamma_{2}
\end{array}\right]\left[\begin{array}{ll}
0 & 1 \\
1 & 0
\end{array}\right]=\left[\begin{array}{cc}
\gamma_{12} & \gamma_{1} \\
\gamma_{2} & 0
\end{array}\right]=T^{\prime}
$$


By Lemma 2.15 there exists a matrix $V=\left[\begin{array}{ll}v_{11} & v_{12} \\ v_{21} & v_{22}\end{array}\right] \in S L(n, \mathbb{H}[s])$ such that

$$
V T^{\prime}=\left[\begin{array}{ll}
v_{11} & v_{12} \\
v_{21} & v_{22}
\end{array}\right]\left[\begin{array}{cc}
\gamma_{12} & \gamma_{1} \\
\gamma_{2} & 0
\end{array}\right]=\left[\begin{array}{cc}
\gamma_{1}^{\prime} & v_{11} \gamma_{1} \\
0 & v_{21} \gamma_{1}
\end{array}\right] \text {, }
$$

where $\gamma_{1}^{\prime}$ is a gcrd of $\gamma_{12}$ and $\gamma_{2}$, and letting $g$ be such that $\gamma_{2}=g \gamma_{1}^{\prime}, v_{21} \sim_{J}$ $g \quad$ and $\left|\operatorname{lc} v_{21}\right|=|\operatorname{lc} g|$. Therefore

$$
\operatorname{Pdet}\left(T P_{12}\right)=\operatorname{Pdet}\left(T^{\prime}\right)=\gamma_{1}^{\prime}{\overline{\gamma^{\prime}}}_{1} v_{21} \gamma_{1} \overline{v_{21} \gamma_{1}}=\gamma_{1}^{\prime}{\overline{\gamma^{\prime}}}_{1} v_{21} \overline{v_{21}} \gamma_{1} \bar{\gamma}_{1}
$$

By Proposition 2.12, $v_{21} \overline{v_{21}}=g \bar{g}$, and thus, since $\gamma_{2} \bar{\gamma}_{2}=g \gamma_{1}^{\prime} \overline{g \gamma_{1}^{\prime}}=\gamma_{1}^{\prime} \overline{\gamma^{\prime}}{ }_{1} g \bar{g}$, by (3.4)

$$
\operatorname{Pdet}\left(T P_{12}\right)=\gamma_{1}^{\prime}{\overline{\gamma^{\prime}}}_{1} v_{21} \overline{v_{21}} \gamma_{1} \bar{\gamma}_{1}=\gamma_{1}^{\prime} \bar{\gamma}_{1} g \bar{g} \gamma_{1} \bar{\gamma}_{1}=\gamma_{2} \bar{\gamma}_{2} \gamma_{1} \bar{\gamma}_{1}=\operatorname{Pdet}(T)
$$

The case where $T \in \mathbb{H}^{n \times n}[s]$ can be treated with basis on the previous one. In fact, if $S=B_{l m}(\alpha), \alpha \in \mathbb{H}[s]$, the proof that $\operatorname{Pdet}(T S)=\operatorname{Pdet}(T)$ is analogous to the $2 \times 2$ case. Moreover, note that $P_{l m}$ can be written as a product of matrices $P_{r(r+1)}$, i.e, matrices that are obtained from the identity by changing consecutive rows. Indeed,

$$
P_{l m}=\left(\prod_{r=l}^{m-1} P_{r(r+1)}\right)\left(\prod_{r=2-m}^{-l} P_{(-r)(-r+1)}\right)
$$

for instance

$$
P_{14}=P_{12} P_{23} P_{34} P_{23} P_{12} .
$$

The proof that $\operatorname{Pdet}\left(T P_{r(r+1)}\right)=\operatorname{Pdet}(T)$ is analogous to the $2 \times 2$ case and the result follows.

Proposition 3.9. Pdet is a polynomial determinant, i.e.,

(i) $\operatorname{Pdet}(R)=0$ if and only if $R$ has not full rank.

(ii) $\operatorname{Pdet}\left(R R^{\prime}\right)=\operatorname{Pdet}(R) \operatorname{Pdet}\left(R^{\prime}\right)$ for all $R, R^{\prime} \in \mathbb{H}^{n \times n}[s]$.

(iii) If $R^{\prime}=B_{l m}(\alpha) R, \alpha \in \mathbb{H}[s]$, then $\operatorname{Pdet}\left(R^{\prime}\right)=\operatorname{Pdet}(R)$.

Proof. ( $i$ ) If $R$ has not full rank and $U R=T$, for some $U \in S L(n, \mathbb{H}[s]$ and $T$ a triangular matrix, then $T$ has not full rank. Therefore one of it its main diagonal elements is zero and hence $\operatorname{Pdet}(T)=0$. On the other hand, let $U R=T$, with $U$ and $T$ as in Definition 3.5. If $\operatorname{Pdet}(R)=0$, then there exists $l=1, \ldots, n$ such that $\gamma_{l}=0$. It is easy to check that this implies that the matrix $T$ has not full rank, which implies that $R$ has not full rank.

(ii) Let $R, R^{\prime} \in \mathbb{H}^{n \times n}[s]$ and $U, U^{\prime} \in S L(n, \mathbb{H}[s])$ be such that

$$
U R=T, \quad U^{\prime} R^{\prime}=T^{\prime},
$$

where $T$ and $T^{\prime}$ are triangular matrices whose main diagonal elements are, respectively, $\gamma_{l}$ and $\gamma_{l}^{\prime}, l=1, \ldots, n$. 
By definition we have

$$
\operatorname{Pdet}(R)=\prod_{l=1}^{n} \gamma_{l} \bar{\gamma}_{l} \quad \text { and } \quad \operatorname{Pdet}\left(R^{\prime}\right)=\prod_{l=1}^{n} \gamma_{l}^{\prime}{\overline{\gamma^{\prime}}}_{l} .
$$

Therefore

$$
\operatorname{Pdet}(R) \operatorname{Pdet}\left(R^{\prime}\right)=\prod_{l=1}^{n} \gamma_{l} \bar{\gamma}_{l} \gamma_{l}^{\prime}{\overline{\gamma^{\prime}}}_{l}=\prod_{l=1}^{n} \gamma_{l} \gamma_{l}^{\prime} \bar{\gamma}^{\prime}{ }_{l} \bar{\gamma}_{l}=\prod_{l=1}^{n} \gamma_{l} \gamma_{l}^{\prime} \overline{\gamma_{l} \gamma_{l}^{\prime}} .
$$

Now, note that by $(3.5)$

$$
U R R^{\prime}=T R^{\prime}=T U^{\prime-1} T^{\prime} .
$$

Let $V \in S L(n, \mathbb{H}[s])$ be such that $T U^{\prime-1}=V \widetilde{T}$, with $\widetilde{T}$ triangular. It follows from Lemma 3.8 that

$$
\operatorname{Pdet}(\widetilde{T})=\operatorname{Pdet}\left(T U^{\prime-1}\right)=\operatorname{Pdet}(T)
$$

moreover by (3.7)

$$
V^{-1} U R R^{\prime}=\widetilde{T} T^{\prime}
$$

Thus, since $V^{-1} U \in S L(n, \mathbb{H}[s])$ :

$$
\operatorname{Pdet}\left(R R^{\prime}\right)=\operatorname{Pdet}\left(\widetilde{T} T^{\prime}\right)=\operatorname{Pdet}(\widetilde{T}) \operatorname{Pdet}\left(T^{\prime}\right),
$$

taking into account that the main diagonal elements of $\widetilde{T} T^{\prime}$ are the product of the main diagonal elements of $\widetilde{T}$ and $T^{\prime}$. Finally, since from $(3.8) \operatorname{Pdet}(\widetilde{T})=\operatorname{Pdet}(T)$, we conclude that

$$
P \operatorname{det}\left(R R^{\prime}\right)=\operatorname{Pdet}(\widetilde{T}) \operatorname{Pdet}\left(T^{\prime}\right)=\operatorname{Pdet}(T) \operatorname{Pdet}\left(T^{\prime}\right)=\operatorname{Pdet}(R) \operatorname{Pdet}\left(R^{\prime}\right) .
$$

(iii) By $($ ii $), \operatorname{Pdet}\left(R^{\prime}\right)=\operatorname{Pdet}\left(B_{l m}(\alpha) R\right)=\operatorname{Pdet}\left(B_{l m}(\alpha)\right) \operatorname{Pdet}(R)$. The result follows since it is obvious that $\operatorname{Pdet}\left(B_{l m}(\alpha)\right)=1$.

Recalling Definition 3.3 of the Dieudonné determinant Ddet, note that if $R$ is a constant matrix, i.e., $R \in \mathbb{H}^{n \times n}$,

$$
\operatorname{Pdet}(R)=[\operatorname{Ddet}(R)]^{2}
$$

Indeed, by Lemma 3.2, there exists a matrix $U \in S L(n, \mathbb{H})$ such that $U R=$ $\operatorname{diag}(1, \ldots, 1, \alpha)$, with $\alpha \in \mathbb{H}$. Hence $\operatorname{Ddet}(R)=|\alpha|$ and $\operatorname{Pdet}(R)=\alpha \bar{\alpha}=|\alpha|^{2}$.

On the other hand, in [9] the Study determinant Sdet of a matrix $R \in \mathbb{H}^{n \times n}$ is introduced as the determinant of its complex adjoint matrix

$$
R^{c}=\left[\begin{array}{cc}
R_{1} & R_{2} \\
-\bar{R}_{2} & \bar{R}_{1}
\end{array}\right]
$$

where $R_{1}$ and $R_{2}$ are complex adjoint matrices such that $R=R_{1}+R_{2} \mathbf{j}$. Since, as shown in [7], $\operatorname{Sdet}(R)=[\operatorname{Ddet}(R)]^{2}$ we conclude that

$$
\operatorname{Sdet}(R)=\operatorname{Pdet}(R) \text {. }
$$

Now, in the polynomial case, defining the complex adjoint $R^{c}(s)$ of a matrix $R(s) \in$ $\mathbb{H}^{n \times n}[s]$ and its Study determinant Sdet $(R(s))$ in the obvious way, it can be shown $[26$, Theorem 3.3.5] that

$$
\operatorname{Sdet}(R(s))=\operatorname{Pdet}(R(s))
$$

which generalizes the relation (3.9) for the polynomial case. 


\section{Eigenvalues}

In this section we show that, as should be expected, the zeros of $\operatorname{Pdet}(s I-A)$ coincide with the right eigenvalues of $A$.

A quaternion $\lambda$ is said to be a right eigenvalue of $A \in \mathbb{H}^{n \times n}$ if $A v=v \lambda$, for some nonzero quaternionic vector $v \in \mathbb{H}^{n}$. The vector $v$ is called a right eigenvector associated with $\lambda$. The set

$$
\sigma_{r}(A)=\{\lambda \in \mathbb{H}: A v=v \lambda, \text { for some } v \neq 0\}
$$

is called the right spectrum of $A$. An efficient algorithm to calculate the right eigenvalues of quaternionic matrices can be found in [27].

The following proposition plays an important role in the proof of Theorem 4.2.

Proposition 4.1. [28] Let $A \in \mathbb{H}^{n \times n}$. Then

$$
\lambda \in \sigma_{r}(A) \Rightarrow[\lambda] \subseteq \sigma_{r}(A),
$$

where $[\lambda]$ denotes the equivalence class of $\lambda$ (cf. page 3).

Theorem 4.2. ${ }^{1}$ Let $A \in \mathbb{H}^{n \times n}$. Then

$$
\lambda \in \sigma_{r}(A) \Leftrightarrow \lambda \text { is a zero of } \operatorname{Pdet}(s I-A) .
$$

Proof. Assume first that $A$ is full rank (invertible). Then, as happens for real matrices, there exists an invertible matrix $S \in \mathbb{H}^{n \times n}$ such that $A^{\prime}=S^{-1} A S$ is a companion matrix, i.e., has the form

$$
A^{\prime}:=\left[\begin{array}{ccccc}
0 & 1 & 0 & \cdots & 0 \\
0 & 0 & 1 & \cdots & 0 \\
\vdots & \vdots & \vdots & \ddots & \vdots \\
0 & 0 & 0 & \cdots & 1 \\
-a_{0} & -a_{1} & -a_{2} & \cdots & -a_{n-1}
\end{array}\right] \in \mathbb{H}^{n \times n}
$$

Note that $\sigma_{r}\left(A^{\prime}\right)=\sigma_{r}(A)$. Indeed, $A^{\prime} v=v \lambda$ for some nonzero $v \in \mathbb{H}^{n}$ if and only if $A^{\prime} v^{\prime}=v^{\prime} \lambda$, where $v^{\prime}=S v$. On the other hand, $s I-A=s I-S A^{\prime} S^{-1}=$ $S\left(s I-A^{\prime}\right) S^{-1}$, which implies that $\operatorname{Pdet}(s I-A)=\operatorname{Pdet}\left(s I-A^{\prime}\right)$. Moreover, it is not difficult to see that there exist matrices $P$ and $Q \in S L(n, \mathbb{H}[s])$ such that

$$
P\left(s I-A^{\prime}\right) Q=\operatorname{diag}(-1, \ldots,-1, d(s))=: D,
$$

with $d(s)=s^{n}+a_{n-1} s^{n-1}+\cdots+a_{1} s+a_{0} \in \mathbb{H}[s]$. Then,

$$
\operatorname{Pdet}\left(s I-A^{\prime}\right)=\operatorname{Pdet}\left(P^{-1} D Q^{-1}\right)=\operatorname{Pdet}\left(P^{-1}\right) \operatorname{Pdet}(D) \operatorname{Pdet}\left(Q^{-1}\right)=d \bar{d} .
$$

\footnotetext{
${ }^{1}$ In [22], Theorem 8.5.1 states that if $d(s) \in \mathbb{H}[s]$ and $A$ is the companion matrix of $d(s)$ then $\lambda \in \sigma_{r}(A)$ if and only if $d(\lambda)=0$. However that result is not true. Indeed, if $d(s)=s^{2}-(\mathbf{i}+\mathbf{j}) s+\mathbf{k}$, the companion matrix of $d(s)$ is $A=\left[\begin{array}{cc}0 & 1 \\ -\mathbf{k} & \mathbf{i}+\mathbf{j}\end{array}\right]$; it turns out that $\mathbf{i}$ is a right eigenvalue of $A$ associated with the eigenvector $v=\left[\begin{array}{c}\mathbf{i}+\mathbf{j} \\ -1-\mathbf{k}\end{array}\right]$, but $d(\mathbf{i})=2 \mathbf{k} \neq 0$. Nevertheless, $\mathbf{i}$ is a zero of $\operatorname{Pdet}(s I-A)=\left(s^{2}+1\right)^{2}$.
} 
Hence it suffices to prove that

$$
\lambda \in \sigma_{r}\left(A^{\prime}\right) \Leftrightarrow \lambda \text { is a zero of } d \bar{d} .
$$

" $\Leftarrow "$ Let $\lambda$ be a zero of $d \bar{d}$. By Proposition 2.6, $\lambda$ is a zero of $\bar{d} d$, i.e., $(\bar{d} d)(\lambda)=0$. If $d(\lambda)=0$, i.e., $\lambda^{n}+a_{n-1} \lambda^{n-1}+\cdots+a_{1} \lambda+a_{0}=0$, it is immediate that $A^{\prime} v=v \lambda$, with $v=\left[\begin{array}{llll}1 & \lambda & \cdots & \lambda^{n-1}\end{array}\right]^{T}$. Hence $\lambda \in \sigma_{r}\left(A^{\prime}\right)$.

On the other hand, suppose that $d(\lambda) \neq 0$. Since, by hypothesis $(\bar{d} d)(\lambda)=0$, by Proposition 2.10 there exists $\lambda^{\prime} \in[\lambda]$ such that $\bar{d}\left(\lambda^{\prime}\right)=0$. This implies, by [21, Theorem 16.4], that there exists $\lambda^{\prime \prime} \in\left[\lambda^{\prime}\right]=[\lambda]$ such that $d\left(\lambda^{\prime \prime}\right)=0$. From the previous case we conclude that $\lambda^{\prime \prime} \in \sigma_{r}\left(A^{\prime}\right)$ which, by Proposition 4.1, implies that $\lambda \in \sigma_{r}\left(A^{\prime}\right)$.

" $\Rightarrow$ " Let $\lambda \in \sigma_{r}\left(A^{\prime}\right)$, i.e., $A^{\prime} v=v \lambda$, for some nonzero $v=\left[v_{1} \cdots v_{n}\right]^{T} \in \mathbb{H}^{n}$. Then

$$
\left\{\begin{array}{l}
v_{2}=v_{1} \lambda \\
\cdots \\
v_{n}=v_{n-1} \lambda \\
-a_{0} v_{1}-a_{1} v_{2}-\cdots-a_{n-1} v_{n}=v_{n} \lambda
\end{array} .\right.
$$

If $v_{1}=0$ it follows from (4.1) that $v_{2}=\ldots=v_{n}=0$ and thus we assume that $v_{1} \neq 0$. Suppose first that $v_{1}=1$. In this case, from (4.1) we have that

$$
\lambda^{n}+a_{n-1} \lambda^{n-1}+\cdots+a_{1} \lambda+a_{0}=0,
$$

i.e., $d(\lambda)=0$. By Proposition 2.9 this implies that $(\bar{d} d)(\lambda)=0$. Suppose now that $v_{1} \neq 1$ and define $\widetilde{v}:=v v_{1}^{-1} \in \mathbb{H}^{n}$ and $\widetilde{\lambda}:=v_{1} \lambda v_{1}^{-1} \in \mathbb{H}$. Note that $\widetilde{v}_{1}=1$ and $\lambda \in[\widetilde{\lambda}]$. Then

$$
A^{\prime} v=v \lambda \Leftrightarrow A^{\prime} v v_{1}^{-1}=v v_{1}^{-1} v_{1} \lambda v_{1}^{-1} \Leftrightarrow A^{\prime} \widetilde{v}=\widetilde{v} \widetilde{\lambda}
$$

and therefore $\widetilde{\lambda} \in \sigma_{r}\left(A^{\prime}\right)$. Analogously to the previous case we conclude that $d(\widetilde{\lambda})=$ 0 . By $[11$, Lemma 4.2] this implies that $(\bar{d} d)(\nu)=0$ for all $\nu \in[\widetilde{\lambda}]$ and hence $(\bar{d} d)(\lambda)=0$.

Suppose now that $A$ is not invertible. Let $V \in \mathbb{H}^{n \times n}$ be a change of coordinates that reduces $A$ to its Jordan form $J=V A V^{-1}$, [13]. By the same arguments as before, it is clear that $\sigma_{r}(J)=\sigma_{r}(A)$ and $\operatorname{Pdet}(s I-J)=\operatorname{Pdet}(s I-A)$. Therefore, we shall assume without loss of generality that $A=J$. Since $A$ is not invertible it will have the block diagonal form $A:=\operatorname{diag}(N, \widetilde{A})$, where

$$
N:=\left[\begin{array}{cccc}
0 & * & \cdots & * \\
0 & 0 & \ddots & \vdots \\
\vdots & \vdots & \ddots & * \\
0 & 0 & \cdots & 0
\end{array}\right] \in \mathbb{H}^{r \times r} \text { and } \widetilde{A} \in \mathbb{H}^{(n-r) \times(n-r)} \text { is invertible. }
$$

Note that $\operatorname{Pdet}(s I-A)=s^{2 r} \operatorname{Pdet}(s I-\widetilde{A})$. Hence, denoting by $\mathcal{N}(M(s))$ the set of zeros of Pdet $M, \mathcal{N}(s I-A)=\{0\} \cup \mathcal{N}(s I-\widetilde{A})$. On the other hand, $\sigma_{r}(A)=$ $\{0\} \cup \sigma_{r}(\widetilde{A})$. Thus, since $\widetilde{A}$ is invertible, it follows from the first part that $\mathcal{N}(s I-\widetilde{A})=$ $\sigma_{r}(\widetilde{A})$, yielding the desired result. 
Corollary 4.3. Let $R(s)=I_{n} s^{m}+R_{m-1} s^{m-1}+\cdots+R_{1} s+R_{0} \in \mathbb{H}^{n \times n}[s]$ and

$$
A=\left[\begin{array}{ccccc}
0 & I_{n} & 0 & \cdots & 0 \\
0 & 0 & I_{n} & \cdots & 0 \\
\vdots & \vdots & \vdots & \ddots & \vdots \\
0 & 0 & 0 & \cdots & I_{n} \\
-R_{0} & -R_{1} & -R_{2} & \cdots & -R_{m-1}
\end{array}\right] \in \mathbb{H}^{m n \times m n}
$$

be the block companion matrix of $R$. Then

$$
\lambda \in \sigma_{r}(A) \Leftrightarrow(\operatorname{Pdet} R)(\lambda)=0 .
$$

Proof. Consider the matrix $(s I-A) \in \mathbb{H}^{m n \times m n}[s]$. It is not difficult to check that, as happens for real matrices, there exist two matrices $P$ and $Q \in S L(m n, \mathbb{H}[s])$ such that $P(s I-A) Q=D$, where $D$ is block diagonal matrix

$$
D:=\operatorname{diag}(-I, \ldots,-I, R(s))
$$

Then, it is clear that

$$
P \operatorname{det}(s I-A)=\operatorname{Pdet}\left(P^{-1} D Q^{-1}\right)=\operatorname{Pdet}\left(P^{-1}\right) P \operatorname{det}(D) P \operatorname{det}\left(Q^{-1}\right)=\operatorname{Pdet}(R) .
$$

Thus, the result follows from Theorem 4.2.

\section{Stability criterion}

With the definition of the polynomial determinant Pdet for quaternionic polynomial matrices given in the previous section, we are now in a position to extend the results on system stability presented in Section 2.1 to the quaternionic case.

Let us first consider a quaternionic state space system

$$
\dot{x}=A x
$$

with $A \in \mathbb{H}^{n \times n}$. The solutions of (5.1) are given by

$$
x(t)=e^{A t} x_{0}, \quad x_{0} \in \mathbb{H}^{n},
$$

[29], where the exponential is defined as usual. If $S \in \mathbb{H}^{n \times n}$ is a change of coordinates that reduces $A$ to its Jordan form $J=S A S^{-1}$, (5.2) can still be written as

$$
\begin{aligned}
& \widetilde{x}(t)=e^{J t} \widetilde{x}_{0}, \quad \widetilde{x}_{0} \in \mathbb{H}^{n}, \\
& x=S^{-1} \widetilde{x} .
\end{aligned}
$$

Taking into account the special structure of the Jordan form, it is possible to prove that the components of $\widetilde{x}(t)$ are given by

$$
e^{\lambda t} p(t)
$$


where the $\lambda$ 's are the elements in the diagonal of $J$ and $p(t)$ is a suitable quaternionic polynomial.

On the other hand, if $\lambda$ is a diagonal element of $J$, there exists a suitable initial value $\widetilde{x}_{0}=E_{r}$ (where $E_{r}$ is the $r$-th vector of the canonical basis of $\mathbb{R}^{n} \subset \mathbb{H}^{n}$ ) such that

$$
\widetilde{x}(t)=e^{\lambda t} E_{r} .
$$

It turns out that the elements in the diagonal of $J$ correspond to the standard ${ }^{2}$ right eigenvalues of $A$. Together with Theorem 4.2, this allows to characterize the stability of $\dot{x}=A x$ in terms of the right spectrum $\sigma_{r}(A)$ of $A$, or equivalently, in terms of the zeros of $\operatorname{Pdet}(s I-A)$.

Proposition 5.1. Let $A \in \mathbb{H}^{n \times n}$. Then the following statements are equivalent.

(i) The quaternionic system described by $\dot{x}=A x$ is stable.

(ii) $\sigma_{r}(A) \subset \mathbb{H}^{-}:=\{q \in \mathbb{H}: \operatorname{Re} q<0\}$

(iii) All the zeros of $\operatorname{Pdet}(s I-A)$ lie in $\mathbb{H}^{-}$.

Proof. The equivalence between ( $i i)$ and (iii) is a direct consequence of Theorem 4.2. $(i) \Rightarrow($ ii $)$ If $\sigma_{r}(A) \not \subset \mathbb{H}^{-}$, there exists a standard right eigenvalue $\lambda$ of $A$ such that $\operatorname{Re} \lambda \geq 0$. Thus, keeping the notation of the previous considerations, for a suitable $r, x(t)=S^{-1} e^{\lambda t} E_{r}$, is a solution of $\dot{x}=A x$. Since, obviously, $\lim _{t \rightarrow+\infty} x(t) \neq 0$ the system is not stable.

$($ ii $) \Rightarrow(i)$ If $\sigma_{r}(A) \subset \mathbb{H}^{-}$, then $\lim _{t \rightarrow+\infty} \widetilde{x}(t)=\lim _{t \rightarrow+\infty} e^{J t} \widetilde{x}_{0}=0$, for all $\widetilde{x}_{0} \in \mathbb{H}^{n}$. By (5.3), this clearly implies that $\lim _{t \rightarrow+\infty} x(t)=0$, for every solution $x(t)$ of $\dot{x}=A x$, proving that the system is stable.

Consider now a quaternionic system described by a higher order matrix differential equation

$$
R\left(\frac{d}{d t}\right) w=0
$$

with $R(s):=R_{m} s^{m}+\cdots+R_{1} s+R_{0} \in \mathbb{H}^{n \times n}[s]$. Assume first that $R(s)$ has full rank. Following the same kind of arguments as in [12] it is possible to show that there exists a unimodular matrix $U(s) \in \mathbb{H}^{n \times n}[s]$ such that

$$
U(s) R(s)=I_{n} s^{m}+\widetilde{R}_{m-1} s^{m-1}+\cdots+\widetilde{R}_{1} s+\widetilde{R}_{0}=: \widetilde{R}(s) .
$$

Defining $x:=\left[\begin{array}{llll}w^{T} & \dot{w}^{T} & \cdots & \left(w^{(m-1)}\right)^{T}\end{array}\right]^{T}$, we obtain the alternative system description

$$
\left\{\begin{array}{l}
\dot{x}=A x \\
w=C x
\end{array}\right.
$$

\footnotetext{
${ }^{2}$ The standard right eigenvalues of a matrix $A \in \mathbb{H}^{n \times n}$ are the complex right eigenvalues of $A$ with nonnegative imaginary part.
} 
with

$$
A=\left[\begin{array}{ccccc}
0 & I_{n} & 0 & \cdots & 0 \\
0 & 0 & I_{n} & \cdots & 0 \\
\vdots & \vdots & \vdots & \ddots & \vdots \\
0 & 0 & 0 & \cdots & I_{n} \\
-\widetilde{R}_{0} & -\widetilde{R}_{1} & -\widetilde{R}_{2} & \cdots & -\widetilde{R}_{m-1}
\end{array}\right] \text { and } C=\left[\begin{array}{llll}
I_{n} & 0 & \cdots & 0
\end{array}\right]
$$

Note that the pair $(C, A)$ is observable, i.e.,

$$
\operatorname{rank}\left[\begin{array}{c}
C \\
C A \\
\vdots \\
C A^{l-1}
\end{array}\right]=l, \quad \text { with } l=n m .
$$

This is equivalent to say that $\left[\begin{array}{c}s I-A \\ C\end{array}\right]$ has a left inverse $[6] V(s)$. Therefore, it follows from (5.5) that

$$
x(t)=V\left(\frac{d}{d t}\right)\left[\begin{array}{l}
0 \\
I
\end{array}\right] w(t) .
$$

Together with the second equation of (5.5) this implies that $\lim _{t \rightarrow+\infty} w(t)=0$ if and only if $\lim _{t \rightarrow+\infty} x(t)=0$. Hence the stability of the original system is equivalent to the stability of (5.5), which in turn, by Corollary 4.3 is equivalent to say that all the zeros of Pdet $R(s)$ lie in $\mathbb{H}^{-}$.

If $R(s)$ has not full rank, Pdet $R(s) \equiv 0$. In this case the corresponding system is not stable, since, (as happens for real systems [12]) $w$ will have some components that can be freely assigned, and hence can be chosen not to tend to zero.

We conclude in this way that stability of (5.4) can be characterized in terms of the zeros of Pdet $R(s)$ as follows.

Theorem 5.2. Consider the system

$$
R\left(\frac{d}{d t}\right) w=0
$$

with $R(s):=R_{m} s^{m}+\cdots+R_{1} s+R_{0} \in \mathbb{H}^{n \times n}[s]$. This system is stable if and only if

$(\operatorname{Pdet} R)(\lambda) \neq 0, \forall \lambda \in \mathbb{H}$ such that $\operatorname{Re}(\lambda) \geq 0$.

\section{Conclusions}

In this paper a definition of determinant for quaternionic polynomial matrices was proposed, which has been inspired by the Dieudonné approach to the nonpolynomial case. Using this new concept, a relationship was established between the right 
eigenvalues of a quaternionic matrix $A$ and the zeros of $\operatorname{Pdet}(s I-A)$ providing a correct formulation for a result suggested by P. M. Cohn [22]. This enabled a characterization of the stability of linear systems with quaternionic coefficients, $R\left(\frac{d}{d t}\right) w=0$, in terms of the zeros of Pdet $(R(s))$, that generalizes the results obtained for the real and complex cases.

\section{A Appendix}

Proof of Proposition 2.12

Suppose first that $a$ and $d$ are monic.

Let $\alpha_{1} \in \mathbb{H}$ be such that $d\left(\alpha_{1}\right)=0$. By Proposition 2.9, $d(s)=\widetilde{d}(s)\left(s-\alpha_{1}\right)$, i.e., $c(s) d(s)=c(s) \widetilde{d}(s)\left(s-\alpha_{1}\right)$, which implies that $(c d)\left(\alpha_{1}\right)=0$. Then, by (2.3), $(a b)\left(\alpha_{1}\right)=0$ but $b\left(\alpha_{1}\right) \neq 0$ because $(b, d)$ are right coprime. Thus, by Proposition 2.10 there exists $\alpha_{1}^{\prime}=b\left(\alpha_{1}\right) \alpha_{1} b\left(\alpha_{1}\right)^{-1} \sim \alpha_{1}$ such that $a\left(\alpha_{1}^{\prime}\right)=0$, i.e., $a(s)=\widetilde{a}(s)\left(s-\alpha_{1}^{\prime}\right)$ for some $\widetilde{a} \in \mathbb{H}[s]$. This implies that $a(s) b(s)=\widetilde{a}(s)\left(s-\alpha_{1}^{\prime}\right) b(s)$. Moreover, by Proposition 2.10

$$
\left(\left(s-\alpha_{1}^{\prime}\right) b(s)\right)\left(\alpha_{1}\right)=\left(\alpha_{1}^{\prime}-\alpha_{1}^{\prime}\right) b\left(\alpha_{1}\right)=0
$$

and hence $\left(s-\alpha_{1}^{\prime}\right) b(s)=\widetilde{b}(s)\left(s-\alpha_{1}\right)$, for some $\widetilde{b} \in \mathbb{H}[s]$. Thus

$$
\begin{aligned}
a(s) b(s)=c(s) d(s) & \Leftrightarrow \widetilde{a}(s) \widetilde{b}(s)\left(s-\alpha_{1}\right)=c(s) \widetilde{d}(s)\left(s-\alpha_{1}\right) \\
& \Leftrightarrow \widetilde{a}(s) \widetilde{b}(s)=c(s) \widetilde{d}(s) .
\end{aligned}
$$

Note that both $\widetilde{a}(s)$ and $\widetilde{d}(s)$ are monic. Proceeding analogously as many times as necessary it is possible to cancel out all the factors of $d(s)$, i.e, if

$$
d(s)=\left(s-\alpha_{m}\right) \cdots\left(s-\alpha_{1}\right), \alpha_{l} \in \mathbb{H}, l=1, \ldots, m,
$$

we obtain $\widehat{a}(s) \widehat{b}(s)=c(s)$, with

$$
\left\{\begin{array}{l}
a(s)=\widehat{a}(s)\left(s-\alpha_{m}^{\prime}\right) \cdots\left(s-\alpha_{1}^{\prime}\right), \alpha_{l}^{\prime} \sim \alpha_{l}, l=1, \ldots, m \\
\left(s-\alpha_{m}^{\prime}\right) \cdots\left(s-\alpha_{1}^{\prime}\right) b(s)=\widehat{b}(s)\left(s-\alpha_{m}\right) \cdots\left(s-\alpha_{1}\right)
\end{array} .\right.
$$

Since $a(s)$ is monic so is $\widehat{a}(s)$. If we prove that $\widehat{a}(s)=1$, by (A.1) and the first equation of (A.2) and as a consequence of Remark 2.7, we have that $a \bar{a}=d \bar{d}$ as desired.

Since $(a, c)$ are left coprime by hypothesis, it is clear that also $(\widehat{a}, c)$ are left coprime, which implies that their conjugates $(\overline{\hat{a}}, \bar{c})$ are right coprime. Moreover,

$$
\widehat{a}(s) \widehat{b}(s)=c(s) \Leftrightarrow \overline{\widehat{b}}(s) \overline{\widehat{a}}(s)=\bar{c}(s) .
$$

In the same way as the factors of $d(s)$ were cancelled out, it is possible to cancel out all the factors of $\bar{c}$ and, letting $\bar{c}(s)=\left(s-\beta_{r}\right) \cdots\left(s-\beta_{1}\right), \beta_{l} \in \mathbb{H}, l=1, \ldots, r$, we 
get $b^{\prime}(s) a^{\prime}(s)=1$, with

$$
\left\{\begin{array}{c}
\bar{b}(s)=b^{\prime}(s)\left(s-\beta_{r}^{\prime}\right) \cdots\left(s-\beta_{1}^{\prime}\right), \beta_{l}^{\prime} \sim \beta_{l}, l=1, \ldots, r \\
\left(s-\beta_{r}^{\prime}\right) \cdots\left(s-\beta_{1}^{\prime}\right) \overline{\widehat{a}}(s)=a^{\prime}(s)\left(s-\beta_{r}\right) \cdots\left(s-\beta_{1}\right)
\end{array} .\right.
$$

By the second equation of (A.3), since $\widehat{a}(s)$ is monic so is $a^{\prime}(s)$. Moreover, $b^{\prime}(s) a^{\prime}(s)=$ 1 implies that $a^{\prime}(s)=1$ and hence also $\widehat{a}=1$, i.e., $\widehat{a}=1$ and thus, as we stated above,

$$
a \bar{a}=d \bar{d} .
$$

Furthermore $a b=c d \Leftrightarrow \bar{b} \bar{a}=\bar{d} \bar{c}$ which, by (A.4), implies that

$$
a b \bar{b} \bar{a}=c d \bar{d} \bar{c} \Leftrightarrow a \bar{a} b \bar{b}=d \bar{d} c \bar{c} \Leftrightarrow a \bar{a}(b \bar{b}-c \bar{c})=0 \Leftrightarrow b \bar{b}=c \bar{c} .
$$

If $a$ and $d$ are not monic, define the quaternions

$$
\mu:=\frac{\overline{a_{n}}}{\left|a_{n}\right|^{2}} \quad \text { and } \quad \nu:=\frac{\overline{d_{m}}}{\left|d_{m}\right|^{2}} .
$$

Then, due to the assumption that $\left|a_{n}\right|=\left|d_{m}\right|$,

$$
\mu \bar{\mu}=\frac{\overline{a_{n}} a_{n}}{\left|a_{n}\right|^{4}}=\frac{1}{\left|a_{n}\right|^{2}}=\frac{1}{\left|d_{m}\right|^{2}}=\nu \bar{\nu} .
$$

Define also the polynomials

$$
\widetilde{a}(s)=a(s) \mu, \quad \widetilde{c}(s)=c(s) \nu^{-1}, \quad \widetilde{b}(s)=\mu^{-1} b(s), \quad \widetilde{d}(s)=\nu d(s) .
$$

Since by hypothesis $(a, c)$ are left coprime and $(b, d)$ are right coprime, also $(\widetilde{a}, \widetilde{c})$ are left coprime and $(\widetilde{b}, \widetilde{d})$ are right coprime. Now

$$
a(s) b(s)=c(s) d(s) \Leftrightarrow a(s) \mu \mu^{-1} b(s)=c(s) \nu^{-1} \nu d(s) \Leftrightarrow \widetilde{a}(s) \widetilde{b}(s)=\widetilde{c}(s) \widetilde{d}(s),
$$

where $\widetilde{a}(s)$ and $\widetilde{d}(s)$ are monic and $\widetilde{a} \sim_{J} \widetilde{d}$, and the result follows then from the first part of the proof.

\section{Proof of Lemma 2.15}

This proof will be based in the Euclidian division algorithm for quaternionic polynomials.

If either $\gamma_{1}=0$ or $\gamma_{2}=0$ the result is easy to check. Let then $\gamma_{1} \neq 0$ and $\gamma_{2} \neq 0$. Assume first that $\operatorname{deg} \gamma_{1} \geq \operatorname{deg} \gamma_{2}$. Then there exist $d_{1}, r_{1} \in \mathbb{H}[s]$ such that

$$
\gamma_{1}=d_{1} \gamma_{2}+r_{1} \text {, with } \operatorname{deg} r_{1}<\operatorname{deg} \gamma_{2} \text { or } r_{1}=0 \text {. }
$$

Thus we may write

$$
U_{1}\left[\begin{array}{l}
\gamma_{1} \\
\gamma_{2}
\end{array}\right]=\left[\begin{array}{l}
\gamma_{2} \\
r_{1}
\end{array}\right]
$$


with $U_{1}=\left[\begin{array}{cc}-d_{1} & 1 \\ 1 & 0\end{array}\right]$

If $r_{1}=0, \gamma_{1}=d_{1} \gamma_{2}$ and therefore $\gamma_{2}$ is a gcrd of $\gamma_{1}$ and $\gamma_{2}$. Then, taking $U=U_{1}$, $u_{21}=g=1$ and the result follows. If $r_{1}$ is nonzero, there exist again $d_{2}, r_{2} \in \mathbb{H}[s]$ such that

$$
\gamma_{2}=d_{2} r_{1}+r_{2}, \quad \text { with } \operatorname{deg} r_{2}<\operatorname{deg} r_{1} \text { or } r_{2}=0 \text {, }
$$

and hence, putting $U_{2}=\left[\begin{array}{cc}-d_{2} & 1 \\ 1 & 0\end{array}\right]$,

$$
U_{2}\left[\begin{array}{l}
\gamma_{2} \\
r_{1}
\end{array}\right]=\left[\begin{array}{l}
r_{1} \\
r_{2}
\end{array}\right]
$$

If $r_{2}=0$, combining (A.5) and (A.7) we have that

$$
\gamma_{1}=\left(d_{1} d_{2}+1\right) r_{1} \quad \text { and } \quad \gamma_{2}=d_{2} r_{1}
$$

and, since $d_{1} d_{2}+1$ and $d_{2}$ are right coprime, this implies that $r_{1}$ is a gcrd of $\gamma_{1}$ and $\gamma_{2}$. Moreover, by (A.6) and (A.8), if $U=U_{2} U_{1}$

$$
U\left[\begin{array}{l}
\gamma_{1} \\
\gamma_{2}
\end{array}\right]=\left[\begin{array}{cc}
1 & -d_{1} \\
-d_{2} & 1+d_{2} d_{1}
\end{array}\right]\left[\begin{array}{l}
\gamma_{1} \\
\gamma_{2}
\end{array}\right]=\left[\begin{array}{c}
r_{1} \\
0
\end{array}\right]
$$

Now, $u_{21}=-d_{2}, g=d_{2}$ and therefore $\mid$ lc $u_{21}|=|$ lc $g_{1} \mid$. If $r_{2}$ is nonzero, proceeding in this way, after a certain finite number $l$ of steps we obtain

$$
U_{l}\left[\begin{array}{l}
r_{l-2} \\
r_{l-1}
\end{array}\right]=\left[\begin{array}{c}
r_{l-1} \\
0
\end{array}\right]
$$

and

$$
\begin{aligned}
& \gamma_{1}=\tilde{g} r_{l-1} \\
& \gamma_{2}=g r_{l-1}, \quad r_{0}=\gamma_{2} .
\end{aligned}
$$

with $\widetilde{g}=d_{1} d_{2} \cdots d_{l}+$ lower order terms (l.o.t) and $g=d_{2} d_{3} \cdots d_{l}+$ l.o.t.

Thus

$$
U\left[\begin{array}{l}
\gamma_{1} \\
\gamma_{2}
\end{array}\right]=\left[\begin{array}{l}
t \\
0
\end{array}\right]
$$

with $U=U_{l} \cdots U_{1}$ and $t=r_{l-1}$. Further, it is not difficult to see that

$$
\begin{aligned}
& u_{21}=(-1)^{l-1} d_{l} \cdots d_{2}+\text { l.o.t } \\
& u_{22}=(-1)^{l} d_{l} \cdots d_{2} d_{1}+\text { l.o.t. }
\end{aligned}
$$

Note that, by (A.9) and (A.10) we can conclude that $t$ is a gcrd of $\gamma_{1}$ and $\gamma_{2}$. Moreover $\mid$ lc $u_{21}|=|$ lc $g \mid$ since $\mid$ lc $u_{21}|=|$ lc $d_{l}|\cdots|$ lc $d_{2}|=|$ lc $d_{2}|\cdots|$ lc $d_{l}|=|$ lc $g \mid$. Now, it still remains to prove that $u_{21} \sim_{J} g$. Clearly $\widetilde{g}$ and $g$ are right coprime and since the matrix $U$ is unimodular its elements $u_{21}$ and $u_{22}$ are left coprime. Moreover, from the equation (A.10) we obtain $u_{21} \gamma_{1}+u_{22} \gamma_{2}=0$, which is equivalent to $u_{21} g_{2}+u_{22} g_{1}=0$. Thus, by Definition 2.11 it follows that $u_{21} \sim_{J} g$. 
The case where $\operatorname{deg} \gamma_{2} \geq \operatorname{deg} \gamma_{1}$ is analogous to the previous one. Indeed, with the following multiplication

$$
\left[\begin{array}{ll}
0 & 1 \\
1 & 0
\end{array}\right]\left[\begin{array}{l}
\gamma_{1} \\
\gamma_{2}
\end{array}\right]=\left[\begin{array}{l}
\gamma_{2} \\
\gamma_{1}
\end{array}\right]
$$

we fall into the previous case, where the expressions for $\gamma_{1}$ and $\gamma_{2}$ in (A.9) as well as the expressions for $u_{21}$ and $u_{22}$ in (A.11) are interchanged. It is clear that the result still holds.

\section{Acknowledgement}

This work was supported in part by Portuguese Science Foundation (FCT-Fundação para a Ciência e Tecnologia) through the Unidade de Investigação Matemática e Aplicações of University of Aveiro, Portugal.

\section{References}

[1] Jack B. Kuipers. Quaternions and rotation sequences. Princeton University Press, Princeton, NJ, 1999.

[2] Awad El-Gohary and Ebrahim R. Elazab. Exponential control of a rotational motion of a rigid body using quaternions. Appl. Math. Comput., 137(2-3):195207, 2003.

[3] Stefano De Leo, Gisele C Ducati, and Celso C Nishi. Quaternionic potentials in non-relativistic quantum mechanics. Journal of Physics A: Mathematical and General, 35(26):5411-5426, 2002.

[4] S. De Leo and G. C. Ducati. Real linear quaternionic differential operators. Comput. Math. Appl., 48(12):1893-1903, 2004.

[5] T. Kailath. Linear Systems. Prentice Hall, Englewood Cliffs, 1980.

[6] Jan Willem Polderman and Jan C. Willems. Introduction to Mathematical Systems Theory: A Behavioral Approach, volume 26 of Texts in Applied Mathematics. Springer-Verlag, Berlin, 1997.

[7] Helmer Aslaksen. Quaternionic determinants. Math. Intelligencer, 18(3):57-65, 1996.

[8] Nir Cohen and Stefano De Leo. The quaternionic determinant. Electron. J. Linear Algebra, 7:100-111 (electronic), 2000.

[9] Eduard Study. Zur Theorie der linearen Gleichungen. Acta Math., 42:1-61, 1920.

[10] Jean Dieudonné. Les déterminants sur un corps non commutatif. Bull. Soc. Math. Fr., 71:27-45, 1943. 
[11] Ricardo Pereira, Paula Rocha, and Paolo Vettori. Algebraic tools for the study of quaternionic behavioral systems. Linear Algebra and its Applications, 400:121-140, 2005.

[12] Jan C. Willems. Paradigms and puzzles in the theory of dynamical systems. IEEE Trans. Automat. Control, 36(3):259-294, March 1991.

[13] Zhuoyuan Y. Zhang. An algebraic principle for the stability of difference operators. J. Differential Equations, 136(2):236-247, May 1997.

[14] Susanne Pumplün and Sebastian Walcher. On the zeros of polynomials over quaternions. Comm. Algebra, 30(8):4007-4018, 2002.

[15] Nathan Jacobson. The Theory of Rings. American Mathematical Society, New York, 1943.

[16] Ivan Niven. The roots of a quaternion. Amer. Math. Monthly, 49:386-388, 1942.

[17] Samuel Eilenberg and Ivan Niven. The "fundamental theorem of algebra" for quaternions. Bull. Am. Math. Soc., 50:246-248, 1944.

[18] B. Gordon and T. S. Motzkin. On the zeros of polynomials over division rings. Trans. Amer. Math. Soc., 116:218-226, 1965.

[19] R. Serôdio, E. Pereira, and J. Vitória. Computing the zeros of quaternion polynomials. Comput. Math. Appl., 42(8-9):1229-1237, 2001.

[20] H. C. Lee. Eigenvalues and canonical forms of matrices with quaternion coefficients. Proc. Roy. Irish Acad. Sect. A., 52:253-260, 1949.

[21] T. Y. Lam. A first course in noncommutative rings. Springer-Verlag, New York, 1991.

[22] P. M. Cohn. Skew fields, volume 57 of Encyclopedia of Mathematics and its Applications. Cambridge University Press, Cambridge, 1995.

[23] A. Cayley. On certain results relating to quaternions. Philosophical Magazine, 26:141-145, 1845.

[24] E. H. Moore. On the determinant of an hermitian matrix of quaternionic elements. American M. S. Bull., 28:161-162, 1922.

[25] I. Gelfand and V. Retakh. Quasideterminants. I. Sel. Math., New Ser., 3(4):517-546, 1997.

[26] Ricardo Pereira. Quaternionic Polynomials and Behavioral Systems. PhD thesis, University of Aveiro, 2006. http://193.136.81.248/dspace/bitstream/2052/134/1/TesePhDRicardoPereira.pdf.

[27] Angelika Bunse-Gerstner, Ralph Byers, and Volker Mehrmann. A quaternion QR-algorithm. Numer. Math., 55(1):83-95, 1989. 
[28] J. L. Brenner. Matrices of quaternions. Pacific J. Math., 1:329-335, 1951.

[29] M. Hazewinkel, J. Lewis, and C. Martin. Symmetric systems with semisimple structure algebra: the quaternionic case. Systems Control Lett., 3(3):151-154, 1983. 